\title{
Le potentiel de la géothermie peu profonde dans la transition énergétique
}

\author{
Alain DASSARgUES \\ Hydrogéologie \& Géologie de l'Environnement, Urban \& Environmental Engineering Unit, \\ Université de Liège, B52/3 Sart Tilman, 4000 Liège.
}

\section{Résumé}

Traditionnellement, seule la géothermie profonde était envisagée. Actuellement, les différentes formes de géothermies peu profondes et de faible enthalpie sont plus accessibles car elles présentent moins de risques financiers et environnementaux. Leur multiplication, notamment pour couvrir les besoins énergétiques de (gros ou nombreux) bâtiments, permet à ces systèmes de participer pleinement à la transition énergétique en cours. Ces systèmes géothermiques peu profonds ne requièrent aucune anomalie du gradient géothermique local. Néanmoins, lorsque des pompages et réinjections sont envisagés dans des aquifères peu profonds, les conditions hydrogéologiques influencent fortement l'efficacité (rendement) et la durabilité des systèmes installés mais aussi leur éventuel impact sur la quantité et la qualité des ressources en eaux souterraines. Les différents types de systèmes géothermiques peu profonds sont brièvement présentés, puis l'accent est mis sur les systèmes par doublets en aquifère et sur les anciennes mines.

Mots clés : eaux souterraines, géothermie, transfert de chaleur

\begin{abstract}
Traditionally, only deep geothermal energy systems were considered. Currently, the various forms of shallow geothermal energy of low enthalpy are more accessible because they present fewer financial and environmental risks. Their multiplication, in particular to cover the energy needs of (large or numerous) buildings, allows these systems to participate fully in the ongoing energy transition. These shallow geothermal systems do not require any local geothermal gradient anomalies. Nevertheless, when groundwater pumping and re-injection are considered in shallow aquifers, very specific hydrogeological conditions are required. The latter closely affect the efficiency and sustainability of the systems, but also their possible impact on the quantity and quality of groundwater resources. The different types of shallow geothermal systems are briefly presented, more emphasis is given on pumping-reinjection doublet systems used on shallow aquifers and in old mining works.
\end{abstract}

Keywords: underground waters, geothermal energy, heat transfer

\section{Introduction}

L'accroissement considérable de l'intérêt porté aux énergies renouvelables et en particulier aux systèmes de géothermie peu profonde, nécessite la quantification des transferts de chaleur dans les zones partiellement et complètement saturées du sous-sol. Pour chaque site envisagé, des valeurs précises pour les propriétés/paramètres du transport de chaleur sont 
à quantifier pour concevoir des réservoirs géothermiques et/ou des systèmes de stockage de chaleur dans les aquifères. Ces valeurs ont une double importance car elles influenceront les performances des systèmes géothermiques à court, moyen et long terme, ainsi que les impacts estimés sur les ressources en eaux souterraines. Auparavant, seules les ressources géothermiques profondes étaient prises en compte, mais actuellement, les systèmes géothermiques peu profonds à très faible enthalpie sont considérés presque partout associés à des pompes à chaleur et pour le stockage de chaleur. La géothermie profonde impliquant de hautes températures ne sera pas abordée ici. Cette forme de géothermie existe depuis très longtemps et fait l'objet de nombreux ouvrages de recherche spécifiques.

\section{Transfert de chaleur en milieu souterrain}

Dans les terrains géologiques, la matrice solide n'occupe pas l'ensemble de l'Elément de Volume Représentatif (i.e. le volume sur lequel les valeurs des propriétés du milieu sont quantifiées). Dans les sédiments granulaires, il y a des pores entre les grains solides. Dans les roches indurées et plissées, il y a en plus des fractures et des fissures avec des ouvertures variables. Même si les roches sont solides et compactes, elles sont souvent considérées conceptuellement comme partiellement ou totalement poreuses. Dans l'équation de conservation de la chaleur dans un milieu supposé poreux, continu et saturé, les flux de conduction, d'advection/convection et de dispersion de chaleur sont pris en compte (parmi d'autres : Therrien et al. 2010, Klepikova et al. 2016, Dassargues 2018) :

$$
\frac{\partial \rho_{b} c_{b} T}{\partial t}=-\nabla \cdot\left[\rho_{w} c_{w} \boldsymbol{q} T-\left(\lambda_{b}+c_{b} \rho_{b} \boldsymbol{D}\right) \cdot \nabla T\right]+Q_{T}
$$

où $T$ la température $\left({ }^{\circ} \mathrm{K}\right)$ est la variable principale, $\nabla T$ est le gradient de température $\left({ }^{\circ} \mathrm{K} / \mathrm{m}\right)$, $\lambda_{b}$ est la conductivité thermique du milieu $\left(\mathrm{W} /\left(\mathrm{m}^{\circ} \mathrm{K}\right)\right.$ ) (généralement considérée comme un scalaire égal à la moyenne géométrique pondérée des conductivités thermiques du solide et de l'eau $\lambda_{b}=\lambda_{s}^{(1-\theta)} \lambda_{w}^{\theta}$ où $\theta$ est la teneur en eau et $\lambda_{s}, \lambda_{w}$ les conductivités thermiques respectivement du solide et de l'eau), $\rho_{w}$ est la densité de l'eau $\left(\mathrm{kg} / \mathrm{m}^{3}\right), c_{w}$ est la capacité thermique de l'eau $\left(\mathrm{J} /\left(\mathrm{kg}^{\circ} \mathrm{K}\right)\right), \rho_{w} c_{w}$ est la capacité thermique volumique de l'eau $\left(\mathrm{J} /\left(\mathrm{m}^{3}{ }^{\circ} \mathrm{K}\right)\right)$, $q$ est le vecteur de flux total d'eau $(\mathrm{m} / \mathrm{s})$ dû à la loi de Darcy et à l'effet possible de la température sur la densité et la viscosité de l'eau, $\rho_{b}$ est la densité apparente (globale) du 
milieu $\left(\mathrm{kg} / \mathrm{m}^{3}\right), c_{b}$ est la capacité thermique apparente (globale) du milieu $\left(\mathrm{J} /\left(\mathrm{kg}^{\circ} \mathrm{K}\right)\right)$, et $\rho_{b} c_{b}$ est la capacité thermique globale (ou apparente) volumique du milieu $\mathrm{J} /\left(\mathrm{m}^{3 \circ} \mathrm{K}\right), \boldsymbol{D}$ est le tenseur de dispersion thermique dépendant du flux de Darcy et de la dispersivité thermique du milieu $\left(\mathrm{m}^{2} / \mathrm{s}\right), Q_{T}$ est le terme de source $\left(Q_{T}>0\right)$ ou de disparition $\left(Q_{T}<0\right)$ de chaleur. Tous les termes de cette équation (1) sont exprimés en $\mathrm{W} / \mathrm{m}^{3}\left[\mathrm{ML}^{-1} \mathrm{~T}^{-3}\right]$. En divisant par la capacité thermique volumique globale $\left(\rho_{b} c_{b}\right)$, on obtient l'équation suivante :

$$
\frac{\partial T}{\partial t}=-\nabla \cdot\left[\frac{\rho_{w} c_{w}}{\rho_{b} c_{b}} \boldsymbol{q} T-\frac{\lambda_{b}}{\rho_{b} c_{b}} \nabla T\right]+Q_{T} /\left(\rho_{b} c_{b}\right)
$$

où chaque terme est exprimé en ${ }^{\circ} \mathrm{K} / \mathrm{s}$. Dans cette équation (2), le coefficient $\lambda_{b} /\left(\rho_{b} c_{b}\right)$ est le plus souvent appelé diffusivité thermique $(\kappa)$ et sa valeur est de l'ordre de $10^{-7}$ à $10^{-6} \mathrm{~m}^{2} / \mathrm{s}$ :

$$
\kappa=\lambda_{b} /\left(\rho_{b} c_{b}\right)
$$

Le terme $Q_{T} /\left(\rho_{b} c_{b}\right)$ peut aussi être compris comme la température associée au flux unitaire d'eau entrant ou sortant du domaine considéré. Notons que la diffusivité thermique est généralement supérieure de 3 à 4 ordres de grandeur à la diffusion moléculaire d'un soluté dans les eaux souterraines (Anderson 2005). La viscosité dynamique de l'eau diminue avec l'augmentation des températures influençant à la hausse la conductivité hydraulique. Le changement de densité influence moins la conductivité hydraulique que les changements de viscosité, mais la diminution de la densité de l'eau avec des températures plus élevées peut néanmoins créer un écoulement qualifié de 'convection naturelle' ne relevant pas de la loi de Darcy.

\section{Différents types de géothermie}

Les systèmes géothermiques de faible température avec pompe(s) à chaleur ciblent habituellement des couches géologiques peu profondes. Ils permettent de répondre aux besoins de refroidissement et de chauffage de bâtiments et sont généralement appelés 'systèmes de pompe à chaleur sol - eau' (Ground Source Heat Pump - GSHP). A des profondeurs comprises entre 5 et $50 \mathrm{~m}$, le gradient géothermique n'est pas encore vraiment perceptible et les températures sont très stables et proches d'une valeur correspondant à la température locale moyenne annuelle de l'air (i.e. de l'ordre de $11-13{ }^{\circ} \mathrm{C}$ dans nos contrées). 
À plus grande profondeur, le gradient géothermique induit un gradient de température croissant d'environ $0,03{ }^{\circ} \mathrm{C} / \mathrm{m}$.

À très faible profondeur, deux types de systèmes sont développés :

1) des systèmes en boucle fermée avec un fluide caloporteur (constitué d'eau et/ou d'une solution glycolée) circulant dans des tuyaux enterrés situés dans un forage (BTES - Borehole Thermal Energy Storage) ou constituant des boucles sous un jardin, parking, etc.

2) des systèmes ouverts avec des doublets de puits impliquant le pompage et la réinjection d'eau souterraine. Dans ce cas, un aquifère peu profond est utilisé pour l'extraction de chaleur/froid par pompage d'eau souterraine et le stockage de froid/chaleur par injection. Ce type de système peut être considéré comme correspondant à un stockage d'énergie thermique dans un aquifère (ATES - Aquifer Thermal Energy Storage).

À profondeur intermédiaire (entre 150 et 1000m), d'autres systèmes se révèlent efficaces :

3) les puits à colonne permanente (SCW - Standing Column Well) consistent à pomper l'eau souterraine à la base du puits avec une pompe submersible installée à l'intérieur d'un tube vertical crépiné à sa base. L'eau souterraine pompée est alors dirigée (via un échangeur de chaleur intermédiaire) vers le système de chauffage, de ventilation et de climatisation de l'immeuble. Contrairement aux ATES, l'eau souterraine est réintroduite dans le même puits en dessous du niveau piézométrique dynamique mais dans l'espace annulaire autour du tubage servant pour le pompage. Dans les terrains meubles, afin d'éviter les éboulements dans le puits, un tubage externe (en acier ou PVC) est installé. Le volume d'eau contenu dans le puits et son long temps de séjour induisent l'inertie thermique nécessaire à atténuer l'impact des pointes de demande. En période de forte demande, une partie de l'eau pompée peut ne pas être recirculée dans le puits, mais évacuée (parmi d'autres : Banks 2008, Rees et al. 2004, O'Neill et al. 2006). L'optimisation de ces systèmes en fonction d'une demande variable reste complexe (Pasquier et al. 2016).

4) les anciennes mines noyées sont également considérées pour d'éventuelles exploitations géothermiques de faible enthalpie où des circuits ouverts peuvent être organisés avec l'eau chaude pompée (ou injectée) dans les parties profondes du réseau de galeries et l'eau froide réinjectée (ou pompée) dans des anciens travaux moins profonds.

Quel que soit le système géothermique adopté, lorsque la production d'énergie thermique est réalisée via une pompe à chaleur, la puissance $P(\mathrm{en} \mathrm{W})$ peut être exprimée par :

$$
P=\frac{Q \Delta T \rho_{w} c_{w}}{\left(1-\frac{1}{C O P}\right)}
$$


où $C O P$ est le coefficient de performance adimensionnel de la pompe à chaleur égal au rapport entre la puissance utile produite par le système et la puissance électrique consommée, $Q$ est le débit du fluide caloporteur dans la pompe à chaleur $\left(\mathrm{m}^{3} / \mathrm{s}\right), \Delta T$ est la différence de température entre l'amont et l'aval de la pompe à chaleur $\left({ }^{\circ} \mathrm{K}\right)$. En pratique, la puissance $P$ est habituellement exprimée en $\mathrm{kW}$ en prenant l'hypothèse (parfois très simplificatrice) d'une densité de l'eau constante égale à $10^{3} \mathrm{~kg} / \mathrm{m}^{3}$.

Parmi les 4 types de géothermie de basse température, seuls les systèmes par doublets en aquifère et les anciennes mines seront développés ici. Ces systèmes requièrent une compréhension et une quantification approfondie des transferts de chaleur en milieu souterrain pour estimer leur efficacité et leur impact. Les deux autres types de systèmes peuvent également être légèrement influencés par le contexte hydrogéologique dans lequel ils sont implantés mais leurs rendements et impacts sont néanmoins beaucoup plus influencés par les échanges thermiques directement au droit du puits et de son équipement.

\section{Géothermie par doublets en aquifère}

Les systèmes ouverts (ATES) utilisent directement les eaux souterraines comme fluide caloporteur. Ils sont classiquement constitués de doublets de puits de pompage et de réinjection des eaux souterraines pour le chauffage et la climatisation/refroidissement saisonniers (figure 1). Les contextes hydrogéologiques idéaux impliquent une conductivité hydraulique élevée (i.e. permettant un pompage et une réinjection efficaces) avec un gradient piézométrique faible (i.e. limitant le déplacement par advection du panache de différence de température dans l'aquifère). L'emplacement des puits peut être optimisé en fonction des caractéristiques des opérations de pompage/injection nécessaires et de la période de variation considérée (i.e. le plus souvent saisonnière) pour maintenir un bilan énergétique équilibré.

Idéalement, un système ATES devrait perdre le moins d'énergie thermique possible via le panache aval de différence de température, car cela peut induire des effets secondaires sur les écosystèmes dépendant des eaux souterraines. En particulier, dans les zones urbaines, les effets conjoints des multiples utilisations des ressources thermiques et des eaux souterraines peuvent rapidement devenir conflictuelles. Les études d'optimisation doivent être basées sur une compréhension approfondie du système souterrain et sur des données fiables pour les valeurs des paramètres principaux et leur hétérogénéité (Vienken et al. 2015). De nombreux développements ont été publiés récemment sur des techniques de caractérisation efficaces (parmi d'autres: Schwede et al. 2014, Wildemeersch et al. 2014, Wagner et al. 2014, 
Hermans et al. 2015, Klepikova et al. 2016) et impliquant, par exemple, différentes techniques hydro-géophysiques combinées à des tests de traçages impliquant des solutés et de la chaleur. D'autre part, il faut également tenir compte du front d'oxydo-réduction dans les eaux souterraines et pomper soit franchement au-dessus ou alors nettement en-dessous de ce front pour éviter des colmatages dus aux précipitations de $\mathrm{Fe}(\mathrm{OH})_{3}$ (Possemiers et al. 2016).

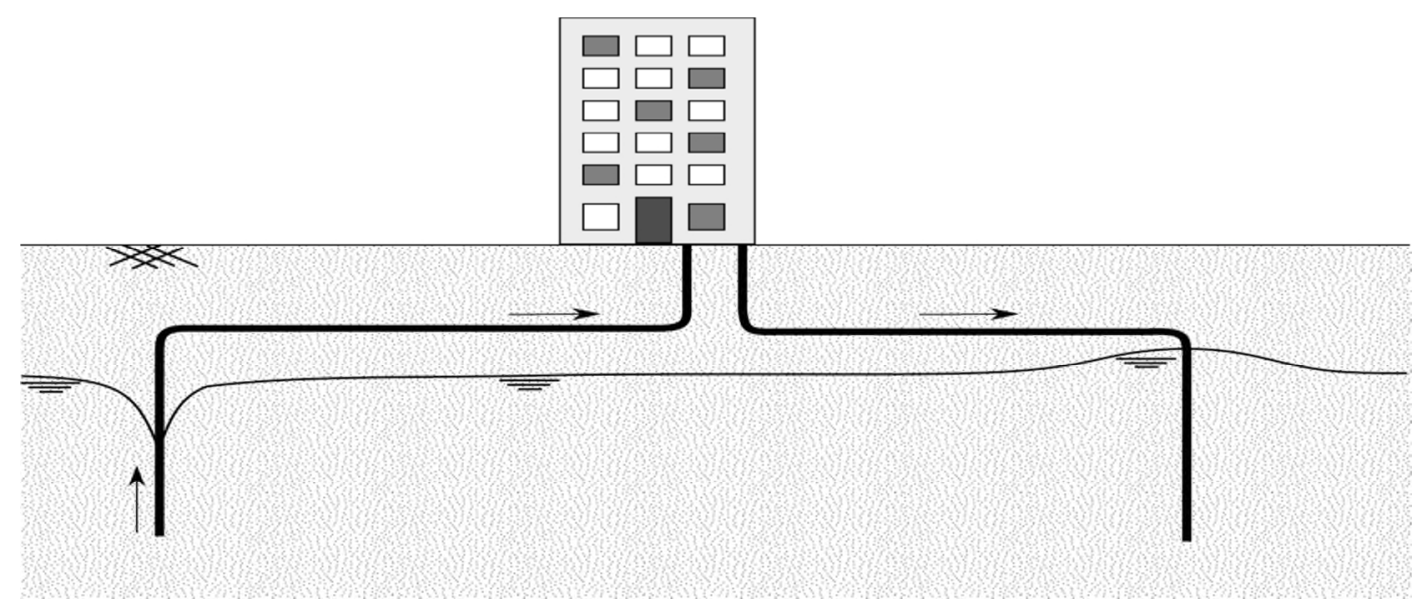

Fig. 1 : Système géothermique en boucle ouverte avec un doublet de puits (modifié d'après Dassargues 2018)

Il est parfois complexe de prévoir l'efficacité des systèmes ATES, notamment en liaison avec l'estimation d'un bilan thermique neutre dans l'aquifère. Le flux de chaleur extrait devrait être équilibré sur une base annuelle, principalement par le flux thermique apporté latéralement par les eaux souterraines et très partiellement par l'influence du flux géothermique venant des couches plus profondes. Les conditions hydrogéologiques spécifiques à un site sont donc le principal facteur d'évaluation de l'efficacité et des impacts d'un système géothermique ATES. Des variations à court terme du stockage et de la récupération d'énergie (i.e. avec des périodes de temps plus courtes que les changements saisonniers) peuvent également être envisagées et simulées. Lorsque le moment et l'ampleur de la demande d'énergie ne coïncident pas avec la production d'énergie renouvelable, un système ATES peut assurer le stockage et pour une récupération de chaleur intra-journalière. Evidemment, ici aussi, un bilan thermique neutre est la garantie d'un impact environnemental limité et d'une probabilité accrue d'efficacité à moyen et long termes. Les ATES étant principalement considérés dans des couches aquifères, les flux de chaleur latéraux par advection sont très importants. Lors de simulations numériques, les conditions de transport de chaleur imposées aux frontières de la zone considérée peuvent donc avoir une influence 
considérable sur les résultats. Ceci est particulièrement vrai dans des conditions d'aquifères captifs, et cela même s'il a été démontré que la conduction thermique à travers les couches peu perméables 'confinant' l'aquifère peut devenir importante (Miotlinski \& Dillon 2015).

Si le bilan géothermique est assuré, l'exploitation de ressources géothermiques peu profondes est généralement considérée comme quasi-parfaitement renouvelable sans compromettre la quantité et la qualité des ressources en eaux souterraines. Cependant, le caractère renouvelable et l'efficacité de ces systèmes peuvent être menacés par des conditions opérationnelles inadéquates et la multiplication de ces systèmes dans des zones urbaines. Les conditions locales de ces systèmes doivent donc être bien identifiées, caractérisées et simulées pour assurer à la fois l'efficacité et la durabilité de l'installation. Ces conditions et d'autres paramètres, tels que la puissance maximale livrée et les coûts de forage, influenceront fortement le choix du système adopté.

\section{Géothermie sur anciennes mines}

Dans les anciennes mines, la cessation des pompages d'exhaure a induit, dans la plupart des cas, l'inondation des anciens travaux. L'écoulement et le transfert de chaleur peut être très rapide dans ce réseau interconnecté et complexe de galeries, de cavités et de puits. A contrario, l'écoulement dans le massif rocheux fracturé et poreux reste un écoulement à faible vitesse. Logiquement, l'eau chaude est pompée (ou injectée) dans une galerie profonde et l'eau froide est réinjectée (ou pompée) dans des anciens travaux moins profonds. Pour évaluer l'efficacité géothermique, il est essentiel de calculer l'évolution de la température dans les zones de pompage en tenant compte du mélange avec les eaux plus froides provenant des parties hautes de la mine (Figure 2). Ceci est particulièrement sensible car des écoulements directs (pipe-like flows) peuvent se produire dans les galeries et les puits ouverts interconnectés. La température de l'eau au sein d'un puits vertical peut être fortement influencée par la convection naturelle résultant des changements de densité de l'eau induits par l'évolution de la température (i.e. le gradient géothermique est lui-même à l'origine du mélange eaux chaudes - eaux froides). Ceci peut induire une chute de température dans la zone de pompage (Hamm \& Bazargan Sabet 2010). Ce mélange est également influencé par les débits de réinjection ainsi que par l'éventuelle variation de salinité des eaux souterraines profondes (i.e. influençant la densité de l'eau). Si seuls les effets thermiques sont pris en compte, un nombre adimensionnel $M$ de convection mixte peut être utilisé : 


$$
M(T)=\left[\rho_{\max }-\rho(T)\right] /\left[\rho(T) \frac{\partial h}{\partial z}\right]
$$

où $\frac{\partial h}{\partial z}$ est le gradient piézométrique vertical. Ce nombre $M$, quantifie l'importance relative de la convection libre verticale par rapport à la convection forcée verticale (ou advection). Il devient grand dans les situations dominées par la convection libre. En pratique, son utilisation est aisée car sa valeur n'est fonction que de la densité maximale prévue de l'eau souterraine $\left(\rho_{\max }\right)$ et du gradient piézométrique vertical. De même, dans une colonne d'eau, un nombre adimensionnel de Rayleigh est défini pour quantifier le rapport entre la convection naturelle et la conduction thermique :

$$
R a=\frac{g \beta(\Delta h)^{3} \Delta T}{v \kappa}
$$

où $\beta$ est le coefficient d'expansion thermique volumique $\left({ }^{\circ} \mathrm{K}^{-1}\right), \Delta h$ est la hauteur de la colonne d'eau dans le puits (m), $\Delta T$ est la différence de température entre la base et le sommet du puits $\left({ }^{\circ} \mathrm{K}\right), v$ est la viscosité cinématique $\left(\mathrm{m}^{2} / \mathrm{s}\right)$, et $\kappa$ est la diffusivité thermale $\left(\mathrm{m}^{2} / \mathrm{s}\right)$. Sa valeur critique pour un puits vertical ou un forage (i.e. supposés à conductivité hydraulique infinie et à parois rigides) est exprimée en fonction du rapport entre le rayon du puits et la hauteur de la colonne d'eau, $\delta=r / \Delta h$ (Love et al. 2007, Hamm \& Bazargan Sabet 2010) :

$$
R a_{c}=\frac{215,6}{\delta^{4}}\left(1+3,84 \delta^{2}\right)
$$

Cette valeur critique montre pourquoi il est recommandé de pomper les eaux souterraines par des puits ou forages de faible diamètre pour éviter que de la convection naturelle vienne s'ajouter à l'advection provoquée par le pompage et la réinjection. Notons que la valeur critique n'est pas fortement diminuée si un support poreux remplace les parois supposées rigides (Love et al. 2007).

Dans nos régions, pour des raisons de sécurité, les vieux puits principaux ont été totalement ou partiellement remblayés par des matériaux hétérogènes et variés, ce qui diminue la conductivité hydraulique à prendre en compte dans le puits. Il est donc plus efficace de réaliser de nouveaux forages qui atteignent directement d'anciennes galeries: galeries profondes pour pomper l'eau chaude et galeries peu profondes pour la réinjection.

Dans tous les cas, les études de faisabilité doivent utiliser des modélisations permettant la simulation d'écoulement à densité variable couplé au transport de chaleur (Fossoul et al. 2011). Les logiciels utilisés doivent permettre une discrétisation spatiale complexe et des 
simulations couplées entre les zones où le transfert de chaleur est rapide (i.e. dans les galeries et puits ouverts) et celles où le transfert est plus lent dans le massif rocheux poreux et fracturé (Dassargues et al. 2019). La paramétrisation et la calibration de ces modèles demande une caractérisation préalable de chaque site étudié.

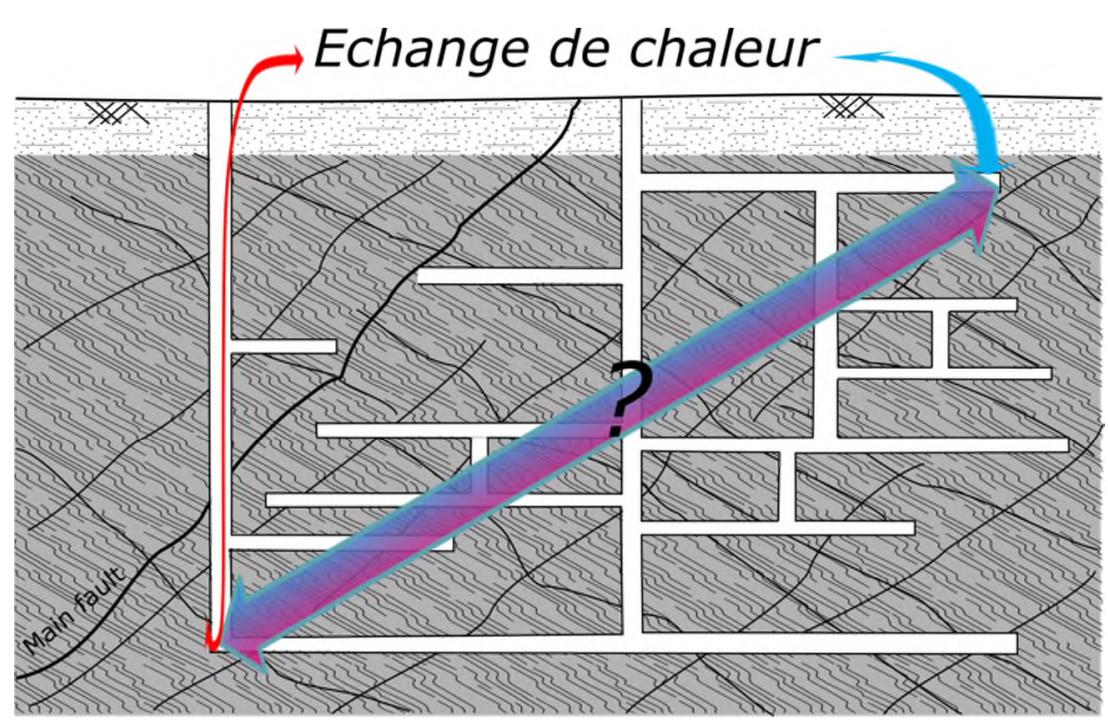

Fig. 2 : Réseau schématique de galeries et puits interconnectés (où un transfert de chaleur rapide est possible) dans un massif rocheux fracturé (modifié d'après Dassargues 2018)

\section{Conclusions}

Le passage des combustibles fossiles aux technologies énergétiques durables exige des changements sociétaux fondamentaux. L'un d'entre eux est l'utilisation de l'eau souterraine pour l'énergie thermique grâce aux systèmes ATES (Aquifer Thermal Energy Storage). Le stockage d'énergie thermique en sous-sol n'est cependant pas une opération facile : les interactions entre les projets de stockage et les eaux souterraines influenceront le dimensionnement en vue d'une efficacité à moyen et à long terme, ainsi que les résultats en termes d'impact et d'évaluation des risques (Kabuth et al. 2017). Ces interactions doivent donc être anticipées sur chaque site. Le récent développement des techniques de mesure, telles que les systèmes DTS (Distributed Temperature Sensing), permettent des 'monitoring' dynamiques produisant des données précises et spatialement distribuées avant l'installation de ces systèmes et pendant leur exploitation. Il s'agit d'optimiser les systèmes pour leur efficacité et leur impact en tenant compte des incertitudes dues à l'hétérogénéité du sous-sol (Hermans et al. 2018, Hoffmann et al. 2019). L'échelle du projet est un facteur important dans 
l'évaluation de sa faisabilité, car le coût des forages et des échangeurs de chaleur avec les systèmes de chauffage et de refroidissement, sont assez élevés. Les délais de retour sur investissement des projets varient entre 2 et 10 ans (Coenen et al. 2010) et il a été constaté qu'ils diminuent généralement avec une forte demande de refroidissement. Pour de gros bâtiments récents, stocker du froid constitue le plus souvent le 'worst case scenario' énergétique. D'autre part, la multiplication des systèmes dans les zones très urbanisées peut induire des influences mutuelles à optimiser.

Dans nos régions, le principal frein à ces systèmes reste néanmoins l'obtention des autorisations de pompage et réinjection. Les régions sont responsables du contrôle et de la gestion des eaux souterraines. Conformément à leurs décrets sur la protection des eaux souterraines, les autorisations ne sont délivrées que dans le respect strict de certaines conditions. Ces règles à respecter pour obtenir les permis sont très influencées par l'idée sousjacente de produire des eaux potabilisables. Ceci est totalement justifié dans les zones où les aquifères sont en effet exploitables pour la distribution publique d'eau potable. Néanmoins, dans beaucoup de zones urbaines ce n'est pas ou plus le cas depuis très longtemps et les conditions devraient être flexibilisées. Un autre problème est l'absence d'un cadre réglementaire clair pour traiter des intérêts conflictuels des différents utilisateurs du sous-sol et du sol.

\section{Références}

Anderson M.P., 2005, Heat as a ground water tracer, Ground Water 43(6) : 951-968.

Banks D., 2008, An introduction to thermogeology: ground source heating and cooling, Blackwell Publishing Ltd.

Coenen L., Raven R. and G. Verbong, 2010, Local niche experimentation in energy transitions: A theoretical and empirical exploration of proximity advantages and disadvantages, Technology in Society 32(4): 295-302.

Dassargues A., 2018, Hydrogeology: groundwater science and engineering, Taylor \& Francis CRC Press, Boca Raton.

Dassargues A., Vopat O. and Ph. Orban, 2019, Geothermal use of old flooded mines: from a risky trial-and-error approach towards challenging predictive simulations. IAH2019 Malaga. Oral paper $n^{\circ} 84$. Proceedings of IAH, p. 781, http://hdl.handle.net/2268/239710 
Fossoul F., Orban P. and A. Dassargues, 2011, Numerical simulation of heat transfer associated with low enthalpy geothermal pumping in an alluvial aquifer, Geologica Belgica $14(1-2) 45-54$.

Hamm V. and B. Bazargan Sabet, 2010, Modelling of fluid flow and heat transfer to assess the geothermal potential of a flooded coal mine in Lorraine, France, Geothermics 39: 177 186.

Hermans T., Wildemeersch S., Jamin P., Orban Ph., Brouyère S., Dassargues A. and F. Nguyen, 2015, Quantitative temperature monitoring of a heat tracing experiment using crossborehole ERT, Geothermics 53 : 14-26.

Hermans Th., Nguyen F., Klepikova M., Dassargues A. and J. Caers, 2018, Uncertainty quantification of medium-term heat storage from short-term geophysical experiments, Water Resources Research 54: 2931-2948.

Hoffmann R., Dassargues A., Goderniaux P. and Th. Hermans, 2019, Heterogeneity and prior uncertainty investigation using a joint heat and solute tracer experiment in alluvial sediments, Frontiers in Earth Science 7: 10.3389/feart.2019.00108

Kabuth A., Dahmke A., Beyer C., Bilke L., Dethlefsen F., Dietrich P., Duttmann R., Ebert M., Feeser V., Görke U.-J., Köber R. Rabbel W., Schanz T., Schäfer D., Würdemann H. and S. Bauer, 2017, Energy storage in the geological subsurface: dimensioning, risk analysis and spatial planning: the ANGUS+ project, Environmental Earth Science 76: 23.

Klepikova M., Wildemeersch S., Jamin P., Orban Ph., Hermans T., Nguyen F., Brouyere S. and A. Dassargues, 2016, Heat tracer test in an alluvial aquifer: field experiment and inverse modelling, Journal of Hydrology 540 : 812-823.

Miotlinski K. and P.J. Dillon, 2015, Relative recovery of thermal energy and fresh water in aquifer storage and recovery systems, Groundwater 53(6) : 877-884.

O’Neill Z.D., Spitler J. and S. Rees, 2006, Performance analysis of standing column well ground heat exchanger systems, ASHRAE Transactions 112, 633-643.

Pasquier P, Nguyen A, Eppner F, Marcotte D and P. Baudron, 2016, Standing column wells. Chapter 10, In: Advances in ground-source heat pump systems : 269-294, Elsevier.

Possemiers, M., Huysmans M., Anibas Ch., Batelaan O. and J. Van Steenwinkel, 2016, Reactive transport modeling of redox processes to assess $\mathrm{Fe}(\mathrm{OH})_{3}$ precipitation around aquifer thermal energy storage wells in phreatic aquifers, Environmental Earth Sciences 75: 648.

Rees S.J., Spitler J.D., Deng Z., Orio C.D. and C.N. Johnson, 2004, A study of geothermal heat pump and standing column well performance, ASHRAE Transactions 110, 3-13. 
Schwede R.L., Li W., Leven C. and O.A. Cirpka, 2014, Three-dimensional geostatistical inversion of synthetic tomographic pumping and heat-tracer tests in a nested-cell setup, Advances in Water Resources 63(0): 77-90.

Therrien R., McLaren R.G., Sudicky E.A. and S.M. Panday, 2010, Hydrogeosphere: A threedimensional numerical model describing fully-integrated subsurface and surface flow and solute transport. Groundwater Simulations Group, University of Waterloo, Waterloo ON.

Vienken T., Schelenz S., Rink K. and P. Dietrich, 2015, Sustainable intensive thermal use of the shallow subsurface - A critical view on the status quo, Groundwater 53(3): 356-361.

Wagner V., Li T., Bayer P., Leven C., Dietrich P. and Ph. Blum, 2014, Thermal tracer testing in a sedimentary aquifer: field experiment (Lauswiesen, Germany) and numerical simulation, Hydrogeology Journal 22(1): 175-187.

Wildemeersch S., Jamin P., Orban Ph., Hermans T., Klepikova M., Nguyen F., Brouyère S. and A. Dassargues, 2014, Coupling heat and chemical tracer experiments for estimating heat transfer parameters in shallow alluvial aquifers, Journal of Contaminant Hydrology 169(0): 90-99. 